\title{
Automatic Fault Localization and Isolation in Power Distribution Network by Decision Support Method
}

\author{
Satya PRAKASH*, Manoj HANS and Vikas THORAT \\ Department of Electrical Engineering, Zeal College of Engineering \& Research, India
}

('Corresponding author's e-mail: sgi.satya@gmail.com)

Received: 25 September 2019, Revised: 26 May 2020, Accepted: 25 June 2020

\begin{abstract}
The power distribution network has grown complex and vulnerable as it increases its demand. The system's reliability has become a prominent factor for the end-users, although the continuity of supply in the distribution network still remains a challenge. In order to achieve the same distribution, automation came into the picture. The term "Distribution Automation" usually refers to an advanced switching system, which works as a subsystem of the existing network. The purpose of the subsystem is to offer real-time observation and control in distribution networks and electricity market operations. Consequently, the development of an autonomous system for isolating failures and restoring power for the distribution of LV (low voltage)/MV (medium voltage) can be an attractive solution for improving energy facilities' reliability. Advanced management techniques are devices and algorithms used to analyze, diagnose, and predict conditions in a distribution network, as well as to identify and take appropriate corrective actions to eliminate, mitigate, and prevent power outages and power quality problems. To demonstrate the model, we used a PIC16F877, CT microcontroller, and a power supply unit.
\end{abstract}

Keywords: Fault Location, Fault Isolation, Distribution Grid, Faulty Feeder, FLISR

\section{Introduction}

The term automation means performing certain tasks automatically, quickly, and accurately without human intervention. Without a microcontroller system, automation cannot be achieved without a communication network and some associated firmware systems. Distribution Automation System (DAS) is an application of automation. Distribution automation systems help automatically to monitor, protect, and control switching behavior in real time mode through intelligent electronic devices. However, with high impedance faults, it makes it challenging to deal with, as discussed by other studies. In many discussions, it has been found that various ranges of filters can be implemented to identify and isolate the faulty section [1-3]. When automated, utilities can segregate and restore power services in the event of a failure in a matter of seconds. As per the Institute of Electrical and Electronics Engineers (IEEE), a Distribution Automation System is defined as a system that allows utilities to monitor distantly, synchronize, and operate distribution components in real-time [4]. Multivariable system is under observation for fault diagnosis achieved by the operation of an emulator. The vector emulator employed in fault detection in combination with Kalam filters has been elaborated [5-7]. New methods are used to monitor basic elements in a timely manner for quick diagnosis and proper response to any event. They also support market pricing, strengthen asset management, and operate efficiently. Automation is part of grid technology. Distribution network technology is used in power generation, transmission, and medium voltage distribution networks.

Automatic control schemes can be presented for detecting the fault automatically, segregation, and recovery in automatic distribution networks. This is called feeder automation [8-10]. If the distribution 
http://wjst.wu.ac.th

network fails, there are two sets of affected end-users. The first group is the bad area of the feeder. They must wait until the end of the process to recover the faulty part before restoring power. The second group consists of end-users who can recover power from the main or backup power source by changing the switch installed in the feeder's appropriate position. Typically, the number of end-users in the second group is much higher than the number of end-users in the first group. The connection of the good and faulty parts to the feeder must be disconnected before restoring the second group's power [11-13]. One can then restore power from the primary or backup power source by changing the switch's correct position on the feeder. In a manual power distribution system, fault isolation and service recovery activities occur after the faulty team detects a fault. However, implementing a feeder automation solution can significantly reduce the disruption suffered by affected end-users [14]. The intelligent feeder automation system automatically detects faults, executes algorithms, separates the faulty part from the normal part, and restores the power to the affected end user as quickly as possible [15]. These technologies rely heavily on four technology areas. For example, monitoring of basic components (sensing and measurement), provision of timely and appropriate response (integrated communication), quick diagnosis of any event (improved interface and intention), and Decision support. [16,17]. Faults occurring in the power system have been differentiated into 2 categories, namely hard and soft types of fault. These faults have been categorized based on the intensity of the fault. State-space model has been implemented for problem detection and isolation [18]. Authors have emphasized the various advantages of the distribution automation system like reduced maintenance, consumer satisfaction, improved data and information, and deferred capital investment. This system's implementation was possible through the existing communication channel and important electrical equipment like voltage regulators, capacitors, and power reclosers $[19,20]$.

\section{Materials and methods}

\section{Proposed system hardware}

This hardware is designed as per the proposed scheme and mentioned specifications below. The hardware has been implemented as per the requirement of the system and based on the simulation. To demonstrate the fault identification on the LV side, 2 different feeders have been considered: namely feeder 1 and feeder 2 .

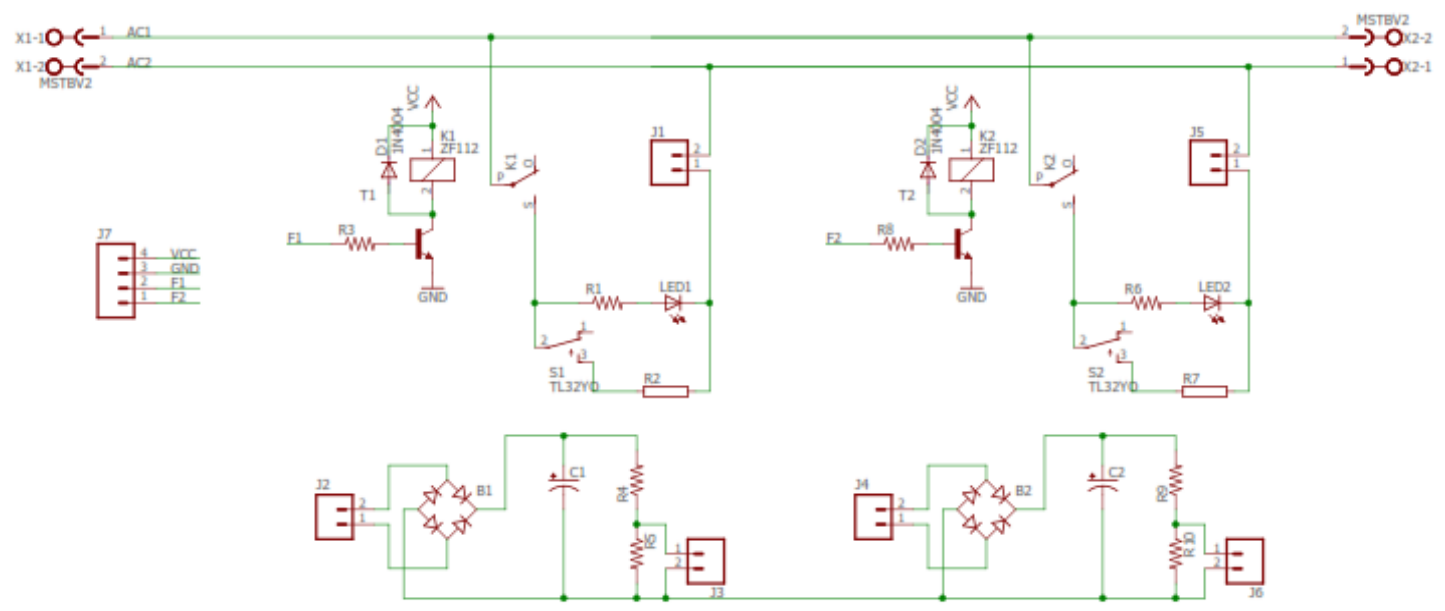

Figure 1 Circuit diagram for proposed system hardware implemented for fault localization and isolation. 


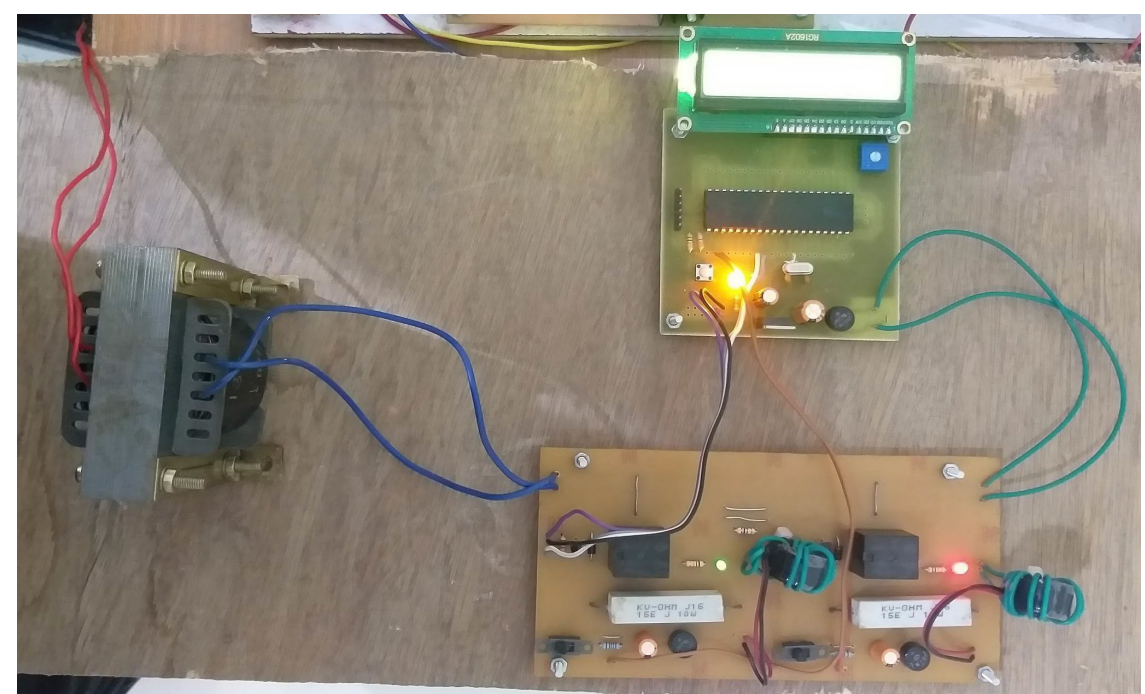

Figure 2 Hardware of proposed system.

\section{Proposed architecture for $\mathrm{LV}$ feeder automaton}

The purpose of the implementation of the proposed hardware is to develop autonomous fault isolation and power recovery algorithm to propose an automatic LV distribution system. Prior to developing the algorithm, literature available was explored to find the possibility of switching from a manual operating system to an automated system. The general form of the LV distribution network is shown in Figure 3. Among them, A and B are HRC fuses, usually closed line jumpers, and usually open line jumpers. In the existing literature of low voltage distribution systems, only 2 switches are called fuses and jumpers, so converting field devices in the low voltage distribution system to remote-controlled switchgear is not complicated. An electrical breaker can be an option to be replaced in place of the fuse. Similarly, isolators can be an option in place of wire jumpers. However, controlling them remotely is a difficult task overall.

Therefore, remote terminal units/smart electronics need to be integrated with these two switches. In order to do so, a microcontroller has been attached to the system. The archive device can operate in standalone mode. The expected automation cannot be achieved by running in standalone mode. These devices must operate as slave devices in the chief control system to achieve automation of the low voltage power supply. Therefore, the main control system needs to be introduced into the automatic isolation of faulty sections and recovery of the system. The main control section must be located at the control center. The device and the main controller need to be integrated with a communication medium. The communication medium may be wired or wireless. A single-line diagram of a typical feeder in a distribution grid is shown in Figure 3. Figure 4 shows the proposed low voltage feeder automation management. The controller and the device are the main parts of the system implemented in hardware. 


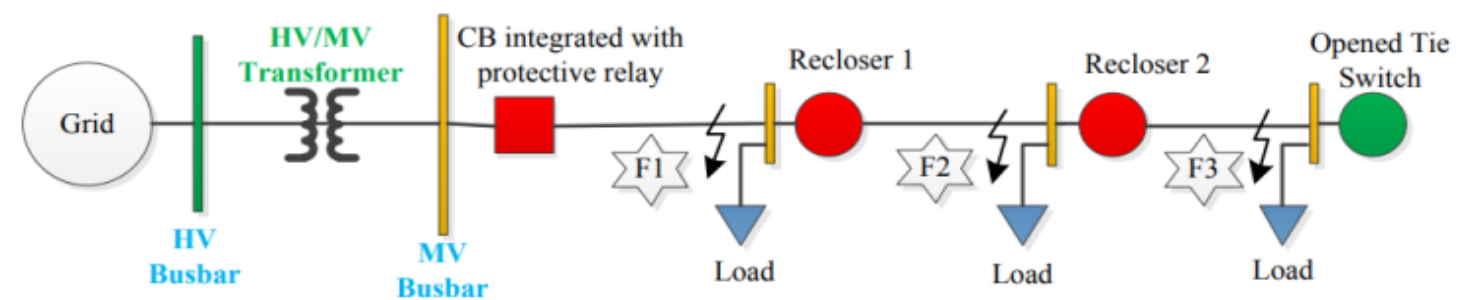

Figure 4 SLD of a Typical Feeder of a Distribution Network.

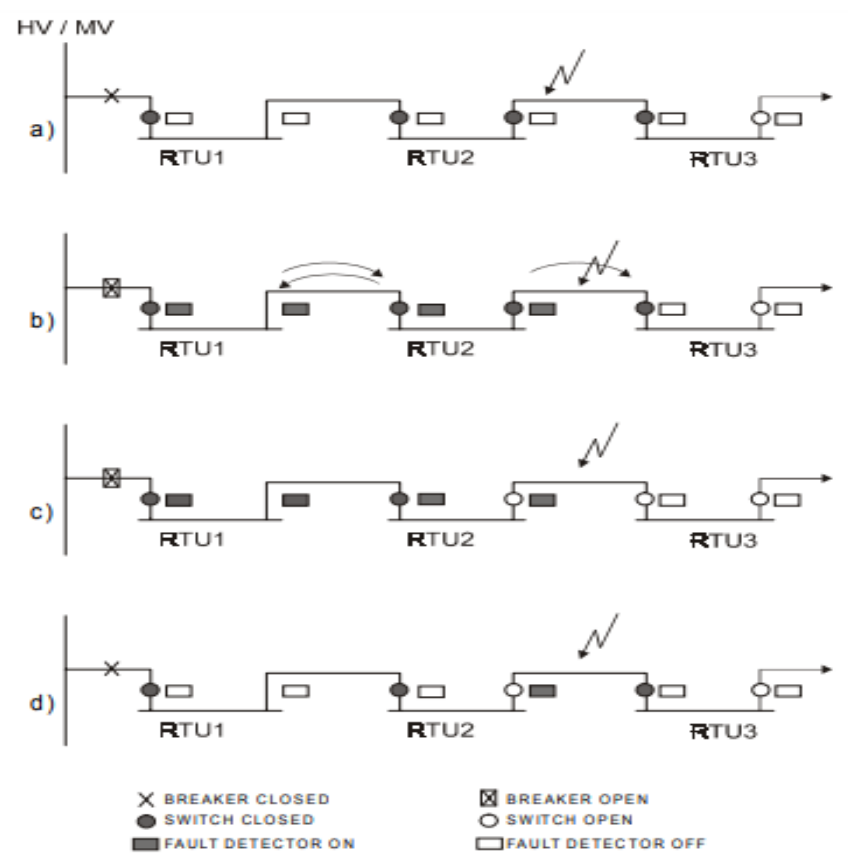

Figure 5 Proposed LV Feeder Automation Management.

Fault location based on the fault current detects the fault portion by calculating the power terminal's fault impedance at a position where the bus voltage is generally less than $80 \%$ of the nominal voltage value. The positive and 0 sequences fault impedance is calculated to determine the fault location based on the distributed network's different grounding systems. The fault location based on the Power Termination Unit (FTU) signals is a typical example to explain this method's operation. As shown in Figure 5, the circuit breaker (CB), the automatic switch 1 (REC1), and the automatic switch 2 (REC2) are placed at the start of the distribution network. The protection coordination algorithm must be considered by setting the tripping and tripping signals of the overcurrent relay.

In the case of underground cables, MV failure management relies on a secondary substation device similar to that described above but supplemented by a fault detector. In this case, the program is somewhat different to avoid closing the defective part. For the operation of the automation system, consider Figure 5. In the event of a fault, initially open the circuit breaker supplying the HV / MV substation. During the line's dead time, the remote terminal unit of substation 1 (RTU 1) sends a message downstream to check the second substation's fault indicator. 


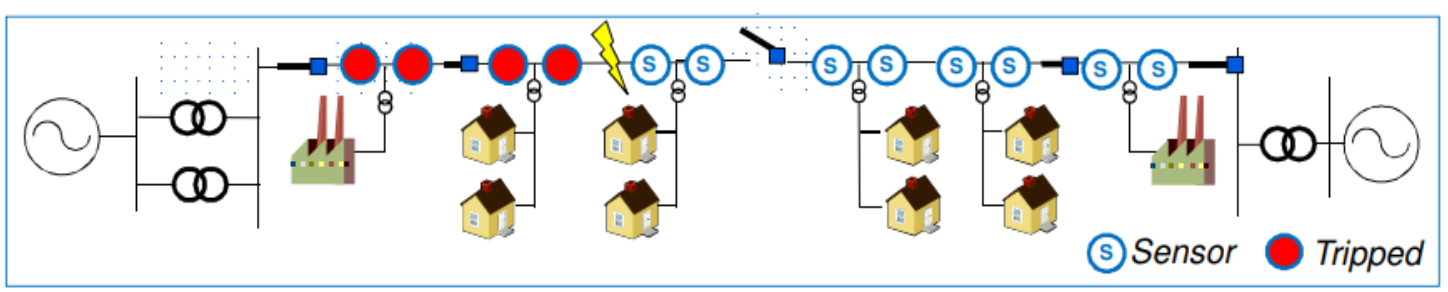

Figure 6 General Fault Current Isolation of LV Feeder.

Figure 6 shows a flow diagram of the control system of the proposed lv feeder automation management and Figure 7 shows general fault current isolation of the LV feeder. In this fault current isolation scheme, consumers connected to the faulty feeder are disconnected from the supply. Hence, supply reliability of other consumers is also affected by the fault.

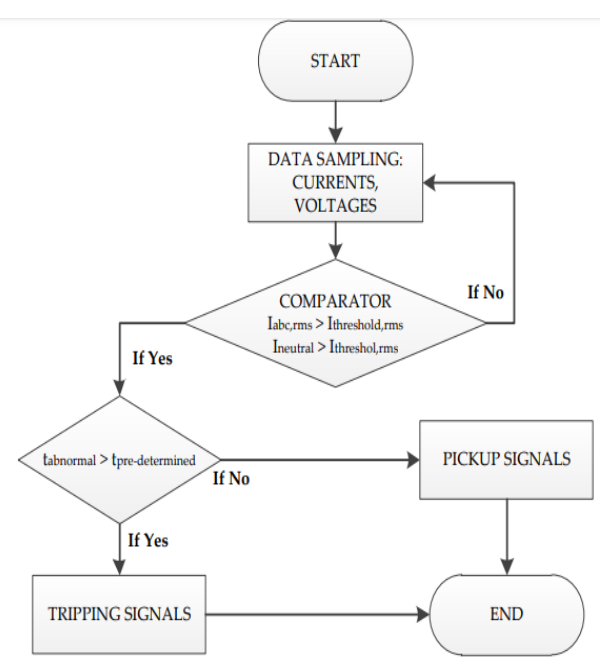

Figure 7 Flow Diagram of Control System of Proposed LV Feeder Automation Management.

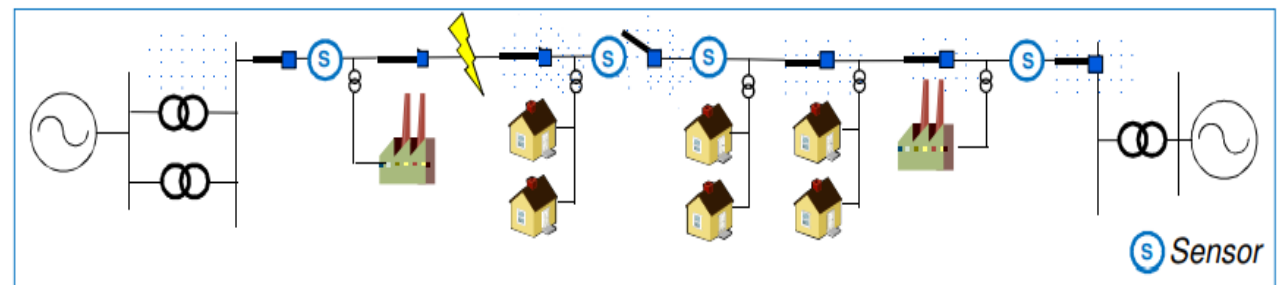

Figure 8 Proposed Scheme of Fault Current Isolation for LV Feeder Automation.

Figure 8 shows the Proposed Scheme of Fault Current Isolation for LV Feeder Automation. In this proposed scheme fault, a current is isolated by removing actual faulty part of feeder, not the entire feeder. So the reliability of consumer supply is more. The hardware and electrical parameters are mentioned below. 


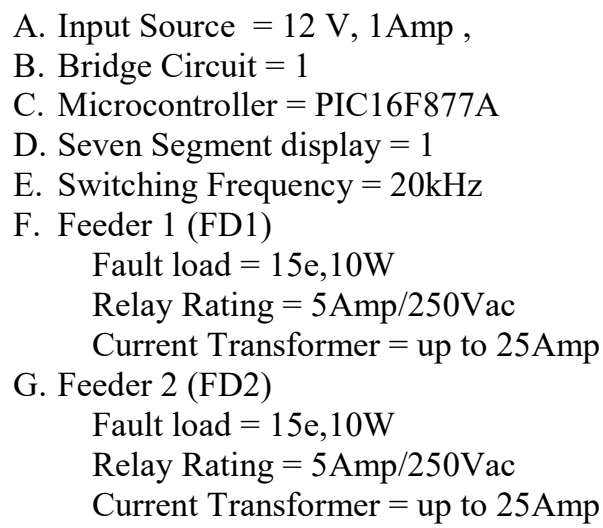

\section{Results and discussion}

The hardware to be implemented was simulated on MATLAB R2018a. The simulation results were observed and noted. The model consists of 2 feeders to demonstrate the switching process in case of fault on one of the given feeder. As there was a fault on feeder 1 at time $t=0.1$ second, feeder 2 gets automatically connected to the load, and the supply is maintained. Figure 9 shows that the current and voltage plot of the Simulink model is observed and found that as the current increases rapidly at time $\mathrm{t}=$ 0.1 second due to fault at feeder 1 . Corresponding to the current at time $t=0.1$, voltage suddenly drops to 0 volt. At the same time, when the current and voltage at feeder 2 were observed, it was found to be zero at time $t=0.1$ second. This clearly suggests that feeder 2 has been isolated from the system when a fault has occurred at feeder 1. Identification and islanding of faulty systems play an important role in providing consumers with reliable, efficient, and safe power supplies. This evolution, combined with the modernization of energy distribution networks, has led to the realization of self-healing capabilities, especially self-healing or automatic FLISR, to improve reliability, quality food, and operational efficiency. By deploying these distribution automation applications, utilities can achieve these performance goals and improve situational awareness and even reduce the potential economic losses. Likewise, by implementing distribution automation applications such as automatic error location, isolation, and service recovery in the event of system failure, the self-healing nature of the distribution network is presented as an effective solution to eliminate errors or interruptions in the distribution network automatically. Therefore, a significant improvement in the reliability of various reliability indexes can be obtained. Reducing "no energy supply" and troubleshooting time, as well as providing "quality service" are other functional benefits. In addition, one can get monetary benefits. Some of them involve increasing revenue (selling more energy), lowering customer costs per failure, and additional revenue for "higher quality" customers, labor/vehicle savings, and more.
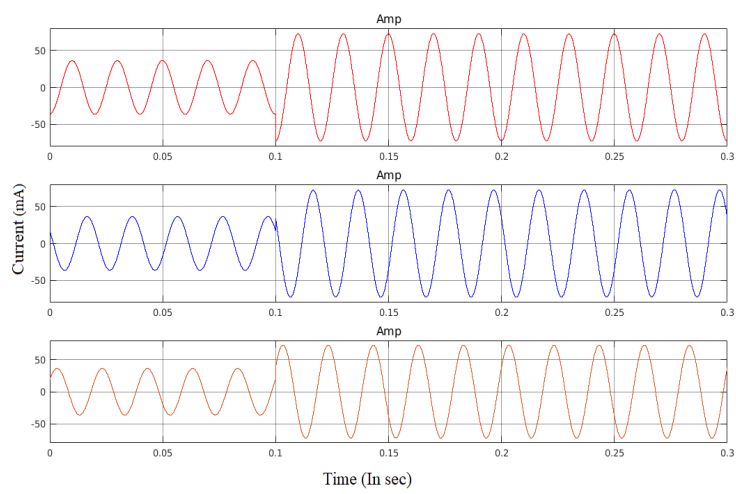

Time (In sec)
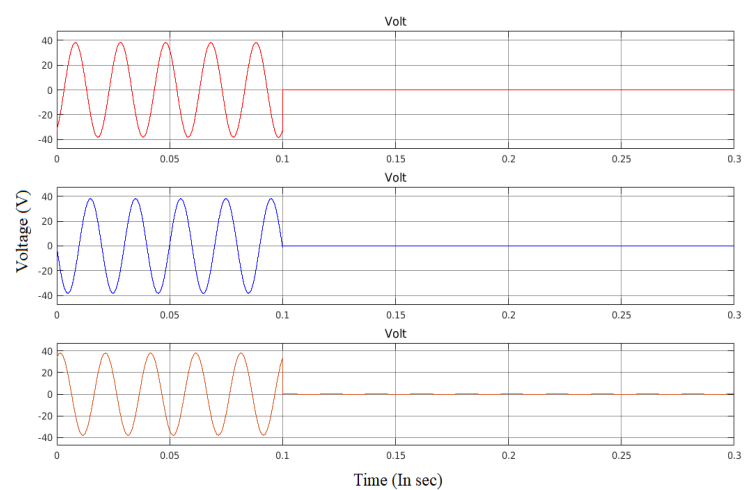

Figure 9 Voltage and current waveform for Feeder 1. 

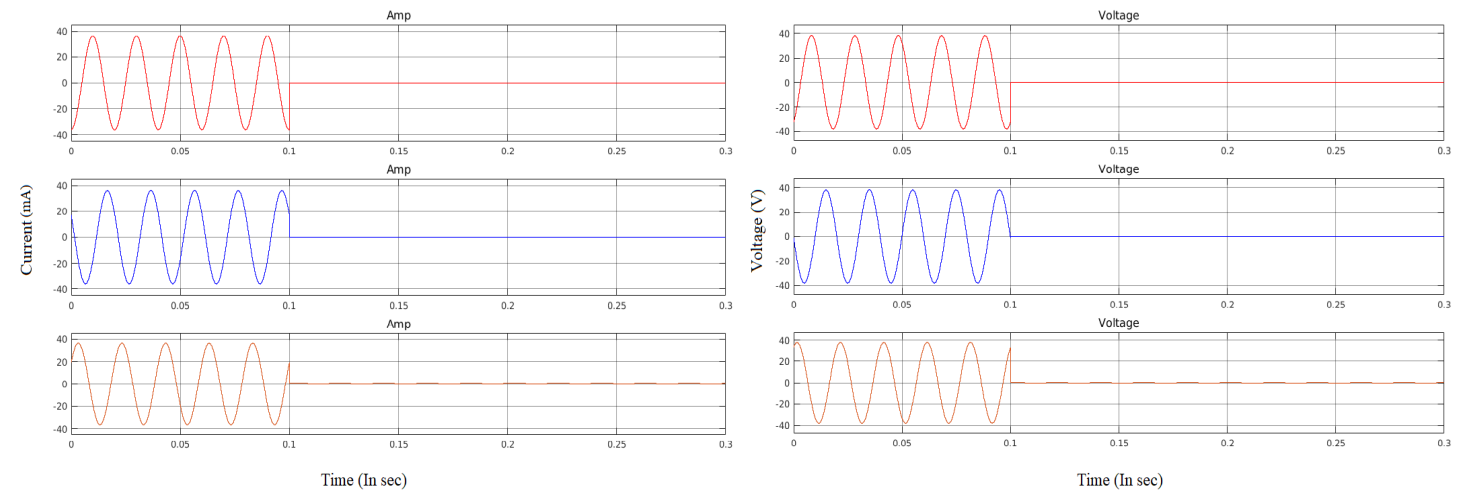

Figure 10 Voltage and current waveform for Feeder 2

Figures 8 - 12 show the current waveforms of feeder 1 and feeder 2 . The voltage waveform of feeder 1 and feeder 2 was obtained by simulating the power system with a fault triggered at $t=0.1 \mathrm{~s}$. A combination of FTU's pick-up and tripping signals and the loss of voltage (LoV) for both fault detection and location was also observed. In Figure 9, the current is increased after a fault on the same feeder when the voltage of that feeder is zero. Thus, this is a combination of FTU's pick-up and tripping signals and the loss of voltage (LoV).

This document contains information about error detection, its location, and isolation in the distribution network. In the current operating policy, if the distribution network fails, the DG unit will automatically disconnect. Disconnection of DG units includes eliminating their effects on fault currents on the power distribution network's conventional protection systems. However, automatically disconnecting the DG unit in the event of a fault reduces the power distribution's reliability and increases the difficulty of fault isolation and service recovery plans. Therefore, the proposed system approach should be extended to be able to adapt to the distribution network in the future reliably.

\section{References}

[1] M Sarwar, F Mehmood, M Abid, AQ Khan, ST Gul and AS Khan. High impedance fault detection and isolation in power distribution networks using support vector machines. J. King Saud Univ. Eng. Sci. 2020; 32, 524-35.

[2] SZ Jamali, SBA Bukhari, O Khan, K Khawaja, M Mehdi, CH Noh and CH Kim. A high-speed fault detection, identification, and isolation method for a last mile radial LVDC distribution network. Energies 2018; 11, 2901.

[3] R Doraiswami and C Lahouari. Fault detection and isolation. Fault Diagnosis and Detection. IntechOpen, London, 2017, p. 534-80.

[4] PM Papadopoulos, L Hadjidemetriou, E Kyriakides and MM Polycarpou. Robust Fault Detection, Isolation, and Accommodation of Current Sensors in Grid Side Converters. IEEE Trans. Indust. Appl. 2016; 53, 2852-61.

[5] M Bollen, R Das, SZ Djokic, P Ciufo, J Meyer, SK Rönnberg and F Zavodam. Power quality concerns in implementing smart distribution-grid applications. IEEE Trans. Smart Grid 2016; 8, 391-9.

[6] V Madani, R Das, F Aminifar, J McDonald, S Venkata, D Novosel, A Bose and M Shahidehpour. Distribution automation strategies challenges and opportunities in a changing landscape. IEEE Trans. Smart Grid 2015; 6, 2157-65.

[7] OK Siirto, A Safdarian, M Lehtonen and M Fotuhi-Firuzabad. Optimal distribution network automation considering earth fault events. IEEE Trans. Smart Grid. 2015; 6, 1010-8. 
http://wjst.wu.ac.th

[8] X Yin, CC Liu, KP Schneider and DT Ton. Toward a resilient distribution system. In: Proceedings of the 2015 IEEE Power \& Energy Society General Meeting, Denver, Colorado, 2015, p. 1-5.

[9] J Liu, H Gao, Z Ma and Y Li. Review and prospect of active distribution system planning. $J$. Modern Power Syst. Clean Energ. 2015; 3, 457.

[10] R Das, V Madani, F Aminifar, JM Donald, SS Venkata, D Novosel, A Bose and M Shahidehpour. Distribution automation strategies: Evolution of technologies and the business case. IEEE Trans. Smart Grid. 2015; 6, 2166-75.

[11] JR Rostron. 2014, High-impedance fault detection and isolation system, U.S. Patent 8,861,155.

[12] S Vimalraj and P Somasundaram. Fault detection, isolation and identification of fault location in low-voltage DC ring bus microgrid system. Int. J. Adv Res Electr. Electron. Instrum. Eng. 2014; 3, 570-82.

[13] S Prakash and SC Gupta. Fuzzy logic based trained fault locating mechanism in power distribution network. Int. J. Emerg. Technol. Adv. Eng. 2012; 7, 2250-459.

[14] A Tanwani, AD Domínguez-García and D Liberzon. An inversion-based approach to fault detection and isolation in switching electrical networks. IEEE Trans. Control Syst. Technol. 2010; 19, 105974.

[15] H Po, R Karki and R Billinton. Reliability evaluation of generating systems containing wind power and energy storage. IET Generat. Transmiss. Distribut. 2009; 8, 783-91.

[16] TS Sidhu and PK Gangadharan. Control and automation of power system substation using IEC61850 communication. In: Proceedings of the 2005 IEEE Conference on Control Applications, Toronto, Canada. 2005, p. 1331-6.

[17] RE Brown, AP Hanson, HL Willis, FA Luedtke and MF Born. Assessing the reliability of distribution systems. IEEE Comput. Appl. Power 2001; 14, 44-9.

[18] JL Aravena and FN Chowdhury. A new approach to fast fault detection in power systems. In: Proceedings of International Conference on Intelligent System Application to Power Systems, Orlando, USA, 1996, p. 328-32.

[19] D Gruenemeyer. Distribution automation: How should it be evaluated? In: Proceedings of the $35^{\text {th }}$ Annual Conference of Rural Electric Power Conference. Dearborn, USA, 1991, p. C3-1.

[20] SL Purucker, RJ Thomas and LD Monteen. Feeder automation designs for installing an integrated distribution control system. IEEE Trans. Power Apparatus Syst. 1985; PAS-104, 2929-34. 